\title{
Profiling the pausing behaviour of EFL learners in real-time computer-aided writing: a multi-method case study
}

\author{
Cuiping Shen ${ }^{1}$ and Ningyang Chen $^{2^{*}}$ (D)
}

\author{
* Correspondence: nychen@suda. \\ edu.cn \\ ${ }^{2}$ School of Foreign Languages, \\ Soochow University, Jiangsu, \\ People's Republic of China \\ Full list of author information is \\ available at the end of the article
}

\begin{abstract}
Technologically enhanced means and devices in language education and research have enabled an in-depth exploration of the dynamics of writing. This study investigated the pausing behaviour of eight Chinese English as a Foreign Language

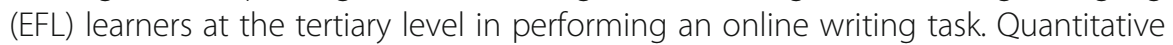
and qualitative data were collected using a combination of methods and techniques, including keystroke logging, screen recording videos, think-aloud protocols, and stimulated recall interviews to establish a profile of each learner's pausing behaviour. The learners' pause profiles were extensively analyzed with a comparative focus on similarities and differences in EFL learners' pausing behaviour across writing skill levels. Overall, the findings revealed a general tendency for the learners to pause most frequently at a low text unit level, i.e., the lexical level. More specifically, lessskilled writers tended to pause more frequently than more-skilled writers at lowerlevel text units, whilst more-skilled writers chose to make more strategic pauses to gain overall control of their writing. Furthermore, these findings help reveal the intricate self-monitoring patterns that undergird individual writer's pausing behaviour and relate these patterns to self-monitoring awareness, writing knowledge and experience, and writing habit. This small-scale multi-method study offers a glimpse into how EFL learners at different skill levels would respond to a real-time online writing task by using resources at their disposal and under conscious monitoring. Methodologically, it adds empirical evidence to previous literature on researching the computer-aided writing process with computer-aided tools and considers productive complementation and triangulation across research approaches and paradigms.
\end{abstract}

Keywords: Pausing behaviour, English as a foreign language (EFL) learner, Real-time writing, Self-monitoring, Computer-aided writing research 


\section{Introduction}

Much is yet to be known about the enigma of writing. Understanding how our mind works when we write has been a topic with lasting significance in language learning and education research since the 1970s and the 1980s (Flower \& Hayes, 1977; Hayes \& Flower, 1987; Reither, 1985; Rohman, 1965). Over past decades, the way we write changes, and with it, the lens through which we are enabled to scrutinize writing processes. Many of these changes can be attributed to the sweeping influence of technology. Developments in second language (L2) online writing, in particular, have led to an interest in diverse new ways of writing shaped by technological advances (GodwinJones, 2018).

This surging interest is also due to the inadequateness of preconceived models that describe writing practices in a traditional sense and that focus exclusively on the end product. On the one hand, cognitive models of writing (e.g., Deane et al., 2008; Flower \& Hayes, 1981; Kellogg, 1996) have been proposed and testified to conceptualize and refine the composition process. On the other, there has been a developing trend for writing instructors to shift their attention from the product to the process (Latif, 2008), who have made efforts to experiment with process-based pedagogies (e.g., Barnhisel et al., 2012; Blyler, 1987; Pandey, 2012). A basic belief underlying this drastic change is that the written text, though readily analyzable, often fails to reveal the full picture of how that piece of writing is constructed - for instance, what procedures are developed, what strategies are used, and what skills are called for. Therefore, by switching the focus onto its process, researchers take an experimental look into the "black box" of writing and begin to understand the complex cognitive activities that contribute to its dynamics.

While most of the experimental efforts are dedicated to examining the writing activity per se, a distinctive line of research turns instead to look at the "non-writing" sessions in the process, i.e., pauses. Trivial as they may seem, pauses play a meaningful part in measuring the speed of language production, as in speech (Laver, 1995) and writing (Wengelin, 2006). As the working memory conceptualization of writing suggests, writers pause when their limited attentional resources fail to sustain the progression of the composition, hence an immediate need to free up resources to deal with the contingent issue that arises to compete against the working memory dedicated to producing the text (Kellogg, 1996, 1999, 2001). In a simplified model, the writing process can be reduced to a process constituted by consecutive episodes of execution intervened by episodes of pauses (Alves et al., 2008; Matsuhashi, 1981; Medimorec \& Risko, 2017; Schilperoord, 2002; Wengelin, 2006). Therefore, pauses as the inactivity periods make an integral part of writing. They are not only practically necessary yet may also contribute strategically to producing a piece of written work.

There are many reasons why we pause in writing. Except for occasional mindwandering, one general reason seems to be that we "get stuck" somehow. While diverse causes can lead to a halt in writing, e.g., a vocabulary crisis, error detection, interrupted train of thought, we are far from an adequate explanation of why and how pauses occur and what they do to our writing. Research is needed to shed more light on this underexplored "inactivity" in writing, given its underestimated presence - we may, in fact, spend more time pausing than actually writing in composition (Medimorec \& Risko, 2017). 
An area of particular interest to researchers and practitioners focuses on pauses in L2 writing; that is, how and why we pause when writing in a language other than our mother tongue. To explore L2 writing pauses, inspirations have been drawn from models that were originally built to account for first language (L1) writing practices (e.g., Flower \& Hayes, 1981; Kellogg, 1996). Studies have shown that L2 writing may not differ fundamentally from L1 writing in terms of cognitive processing, yet compared with L1, the "linguistic encoding processes ... are likely to generate considerable cognitive demands for L2 writers" (Révész et al., 2017, p. 210). How and to what extent L2 writers' performances may yield to such linguistic encoding pressure is one of the issues that the current study intends to explore.

This paper reports a small-scale multi-method study that tracked and recorded the pausing behaviour of eight Chinese English as a Foreign Language (EFL) learners at the tertiary level who undertook an online writing task and completed a series of selfreporting protocols, including think-aloud recordings, post-task interviews and questionnaires. Analysis of a combination of observational and retrospective data established each learner's pause profile. Individual learners' pause profiles were analyzed with a comparative focus on the similarities and differences in learners' pausing behaviours across writing skill levels. After an overview of the studies that inspired the current enquiry, we explain the methodological details of the study design. In the section that follows, we present and discuss our major findings and relate them to previous findings in the literature. This bulk of the paper is followed by further considerations about the limitations and implications of the study as well as the role of technology in changing writing research and instruction.

\section{Review of literature}

Early analysis of writing pauses was largely based on observational data obtained from classroom settings where writing was practiced in the traditional pen-to-paper style. For instance, Matsuhashi (1981) videotaped the writing activity of four high-school students who composed two essays and analyzed their between-word pauses in relation to a set of lexical and syntactical features. Other pioneering efforts to examine pausing behaviours were motivated by the pedagogical interest in exploring the differences between competent and less-competent writers. In a videotape study of beginner and advanced level college students' pausing activities in a 30-min essay writing task (Schumacher et al., 1984), researchers found no difference in the number of pauses (with a 10-s threshold) in each writing session; yet advanced level students tended to undertake more complex cognitive activities during the pause break, resulting in better writing performance in terms of content and organization. Combining evidence from classroom observation with verbal protocol analysis, Raimes (1985) was interested to notice a number of activities that characterized the performances of the less-skilled English as a Second Language (ESL) writers in her class. In particular, she noticed the recursive behaviour of the ESL writers as they went back to read, to consolidate the expression of an idea, and to go forth therefrom; these recursive pausing episodes awaited to be explored in terms of their types, causes, and influences on writing performance (Raimes, 1985).

In computer-assisted writing research, a pause has been operationalized as any short nonscribal period between two keystrokes that lasted longer than a threshold transition 
time (Wengelin, 2006, p. 107). Various criteria and taxonomies have been developed to describe the features of pauses in online writing. For example, Chenu et al. (2014) put forward a temporally driven and a linguistically driven approach: the former designates a threshold for observing the frequency and locations of pauses; the latter places a pause in relation to delimited linguistic boundaries in discourse so as to anchor and analyse their occurrences. Schilperoord (2002) proposed a categorization of pauses on the basis of their cognitive status in discourse production, specifying pauses that signal retrieving, monitoring, and repairing. A binary classification consisting of formulation and revision pauses was adopted in Barkaoui (2019), where the two different types of pauses were distinguished by their subsequent behaviours, i.e., proceeding with the writing or going back to revise. Following Kellogg (1996), Révész et al. (2017) specified pausing during planning, translation, and monitoring subprocesses of writing, and further identified subcategories on the basis of what the pause was intended for: content and organization during planning, lexical retrieval, syntactic encoding, and cohesive devices during translation, while monitoring pauses were more likely to occur at the sentence unit level.

Among these and many more recent studies (e.g., Baaijen et al., 2012; Chan, 2017; Chukharev-Khudilaynen, 2014; Wengelin et al., 2009; Xu, 2018; Zarrabi \& Bozorgian, 2020) that seek to investigate the "higher-level cognitive processes" that underlie writing (Medimorec \& Risko, 2017, p. 1267), a specifical line of empirical work that probes L2 writing pauses has inspired the current study. Following the initial general observation that L2 writers tended to have less global level planning (Dennett, 1985), Moragne e Silva, M. (1988) carried out a six-month case study on one adult writing in L1 (Portuguese) and L2 (English). Data were collected using think-aloud protocols and post-task interviews. The study revealed strong similarities in the individual writer's L1 and L2 writing practices in terms of processes and sub-processes involved in finishing the writing task. Meanwhile, marked differences were detected in the efficiency of attaining specific goals in writing, with less efficient translating practices in L2, possibly hindered by linguistic and other low-level encoding difficulties. Similarly, Spelman Miller's (2000b) study confirmed the L1-L2 differences in writing fluency as manifested in the location and duration of planning pauses. As part of their longitudinal study, Spelman Miller et al. (2008) tracked the English writing performance of 17 Swedish high school students over a period of three years. Using keystroke logging and textual analysis techniques, they found a significant decrease in the number and duration of pauses in students' L2 writing over time, yet they found that the students' writing experience had no significant impact on the length and quality of their written product. With a focus on the role of task complexity in L2 writing practice, Révész et al. (2017) recorded 73 advanced L2 writers' online writing performances via keystroke logging. The quantitative data were complemented by additional stimulated recall comments. The results associated decreased task complexity with improved L2 writing performance: the aided writers tended to pause less, revise more, and produce more complex linguistic structures. These empirical efforts have identified a range of features that characterize L2 pauses in relation to L1 pauses. Since L2 writers are not a homogenous group, more refined and detailed descriptions are needed to reveal the differences between writers at various proficiency and skill levels. 
Of close relevance to the current enquiry are a series of empirical studies conducted in the Chinese context (Nie \& Li, 2016; Xu 2011, 2017, 2018, 2021; Xu \& Qi, 2017). In a case study of two Chinese tertiary-level EFL learners of different skill levels, $\mathrm{Xu}$ (2011) innovated the combined use of keystroke logging and screen recording techniques and discovered noticeable differences in the pausing behaviours between the two writers. The less-skilled writer was found to pause more often yet generally shorter than the more skilled writer. In terms of location, both writers had significantly more pauses before words, and the less-skilled writer had more within-word pauses and before-paragraph pauses than the moreskilled writer. With a larger student sample, Nie and Li (2016) compared the writing pauses made by 30 Chinese English major students and 31 non-English major students in their responses to an online writing task and identified significant differences in pause frequency and duration except before- and afterparagraph pauses. Apart from comparison across skill levels, a genre perspective has been included in a most recent study ( $\mathrm{Xu}, 2021$ ), which compared the keystroke logs of narrative writing and argumentative writing by 60 Chinese sophomore students. The study identified low frequency and long duration planning pauses in writing behaviours across genres, yet the students generally spent more time planning their narrative than their argumentative essays. In writing the former, they were found to make short, frequent pauses, which were largely driven by linguistic encoding, whereas in writing the latter, their pauses were more varied, focusing on content retrieval and organization as well as linguistic encoding. Though some initial endeavour has been made towards a more refined understanding of L2 pauses, empirical evidence is still lacking as to how learners with different backgrounds would proceed with their writing task and what they are actually doing during the writing pauses.

One of the unsolved key concerns is the cognitive mechanism involved in writing pauses, which, though has been interpreted variously based on analysis of log files, defies a definite objective measurement. That is, what the writer is cognitively busy with during the pausing episode may not be readily interpretable without a better understanding of the writer as the cognitive agent engaged in the writing activity. Therefore, to supplement a "subjective" "insider" perspective of the pausing behaviour, the current study attempts to conceptualize writing pauses based mainly on a qualitative analysis of multiple sources of writers' self-reported data. A tripartite model involving observational, evaluative, and diagnostic pauses is developed for profiling the characteristics of pausing behaviours among EFL writers. For a refined picture of how learners at different skill levels behave in an online writing task, the present study focuses on addressing the following three questions:

1. How often, how long, and where do EFL learners pause in online writing?

2. Do EFL learners with different levels of L2 writing skill have similar or different pausing behaviours in online writing? What are these similarities and/or differences?

3. What can these pausing behaviours tell about learners' self-monitoring patterns in online writing? 


\section{Methods}

A multi-method design that combined keystroke logging with additional retrospective and observational measures (Leijten \& Van Waes, 2013) was adopted to investigate the pausing behaviour of EFL learners in real-time computer-assisted writing. Each individual participant's writing process was recorded, charted, and analyzed to establish a pause profile for his/her online writing performance. Multiple data sources were interrogated to identify features of EFL learners' pausing behaviour in online writing and for shared and different tendencies among learners at two different skill levels. Despite its limited scale, the study is along a line of worthy explorations into the cognitive activities during the non-writing sessions. This endeavour intends to shed light on this less well-understood procedure by closely tracking a couple of EFL learners' online writing behaviours. Methodologically, the current study experiments with a multiple-method design which aims at a comprehensive understanding of individual writer's writing activity and the cognitive process involved in this activity. The methods used in the current study can be classified following Janssen et al. (1996), as illustrated in Fig. 1.

On the horizontal axis, direct observations were made of participants' writing activities by the use of concurrent think-aloud protocols and retrospective comments obtained by stimulated recall interviews and post-task questionnaires. Indirect data were collected by software (i.e., keystroke logging and screen recording) and by hand (i.e., observational notes and text analysis). On the vertical axis, synchronous data simultaneously recorded participants' real-time writing behaviours via think-aloud protocols, keystroke logging, screen recording, and researcher's notes taken during the writing task. Asynchronous data, on the other hand, were collected after the writing practice, with stimulated recall interviews prompted by screen-recording videos, subsequent questionnaires, and the writing sample produced in the task. The collection of data from diverse sources had the strength of yielding more valid and better triangulated interpretations about the real-time writing process than relying on one dimension of sources alone.

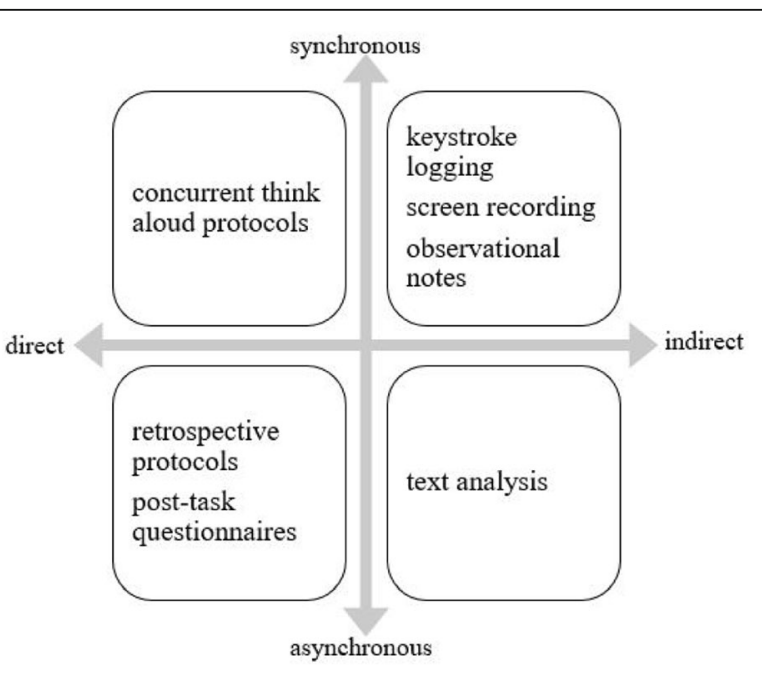

Fig. 1 Classification of methods used in the current study (based on Janssen et al., 1996) 


\section{Participants}

The current study is part of an ongoing research project investigating the online writing practice of Chinese EFL learners. A total of 24 participants (numbered S1-S24) were recruited from first-year full-time undergraduate students at a university in an eastern coastal city of China. The students aged between 18 and 19 at the time of data collection and had 10-12 years of EFL education in the Chinese educational context. These students were assigned to three English classes based on their proficiency levels as estimated by their scores in the pre-college English placement test. They had been taking an English for General Academic Purposes course for one semester taught by the same instructor. As part of the course requirement, the students were expected to compose and submit all their writing assignments online. Thus, the students had online writing experience prior to the study and were familiar with the physical environment of the writing task. After explaining the purpose and requirement of the study, the researchers promised monetary rewards with individually-tailored feedback to motivate the participants. All 24 students confirmed their voluntary participation by signing informed consent.

\section{Procedures}

All participants received a week-long training for thinking aloud techniques (Green, 1998) and online writing with Inputlog. Two students (one male, one female) randomly selected from the 24 students took part in a pilot study that aimed to test and modify the multi-method design and to draft a preliminary coding scheme. The other 22 students were included for data screening. Their real-time computer-aided writing data were collected in a timed online writing task. In the task, each participant was given 30 min (participants would receive a "Time's up" reminder after a lapse of $30 \mathrm{~min}$; yet to ease the tension, participants who did not finish their writing were allowed a maximum of 10 extra minutes) to write on a given topic: facing difficulties. Five minutes were allowed for pre-writing preparation, during which participants could use pen and paper to draft their ideas. The recommended length of writing was around 150 words. Content support was given in the form of three additional statements which could serve as possible topic sentences for structuring paragraphs: (a) life is full of difficulties; (b) some people easily yield to difficulties; (c) one should face difficulties with a positive attitude. During their writing sessions, participants were allowed to use electronic dictionaries yet were disallowed to visit any website. To ensure comprehension, both the content support and the directions were presented in Mandarin Chinese.

Participants' real-time writing activities and think-aloud protocols were simultaneously recorded using pre-installed tools. Each participant's online writing activity was monitored and recorded continuously using keystroke logging and screen recording software. Inputlog 7.0 was used for keystroke logging (Leijten \& Van Waes, 2012), and Camtasia 9.0 (software developed by TechSmith $($ ) was used for on-screen activity recording. Upon finishing the writing task, the participant exited Inputlog and Camtasia and saved the keystroke logging and the screen-recording files. Then the researcher restarted Camtasia and invited the participant to watch the Camtasia video, which served as a stimulus for the participant's recall of the writing process. During the retrospection, the researcher referred to the observational notes and interviewed the 
participant about the hesitations and pauses that were left unexplained in the thinkaloud account. Typical queries included: "Why did you pause here?" "What were you thinking at that time?" Immediately after the interview, the participant completed a post-task questionnaire, which assessed his/her perceptions of (a) overall mental effort exerted, (b) pre-writing planning activities, (c) difficulties and challenges in online writing and solutions, (d) awareness of noticing problems and correcting mistakes in writing, and (e) overall evaluation of the participant experience.

The entire data collection process lasted for around $90 \mathrm{~min}$ for each participant, who took part in the study separately, in an undisturbed place of their preference, e.g., the university computer lab or teacher's office. Efforts were made to naturalize the data collection process and to keep participants actively engaged yet comfortably relaxed.

After collecting the data, we invited two EFL teachers to rate participants' writing samples (see Appendix 3). Both teachers had over 10 years of teaching experience and the experience of rating the writing section in College English Test (CET), a national EFL test in China for non-English major undergraduate students, yet neither had the experience of working with the student participants in the study. The teachers received training before the rating and practiced rating writing samples of varying qualities. The rating rubric (Appendix 1) was adapted from that used in CET band 4, which adopted a holistic rating format. All writing samples were numbered following the alphabetical order of the authors' Chinese names to avoid the influence of an order effect (Green, 1998 ) in scoring. Both teachers rated the writings separately. Inter-rater reliability was calculated using Pearson correlation coefficients and reached a high score after solving the discrepancies via discussion, $r(22)=0.95$. The grouping criteria were taken from a comparable previous study (Xu \& Ding, 2014) that adopted precisely the same rating rubric to assess Chinese EFL students' online writing samples. As such, four students who ranked the top, with a score of 12 or above out of a total score of 15 points, formed the more-skilled EFL writer group in the study; whereas the four students ranking at the bottom, with a score of 8 or below, formed the less-skilled group. These eight students constituted the focused cases in the current study (Table 1).

\section{Data coding and analysis}

The keystroke log files were analyzed in terms of pausing behaviours. For comparability with previous measures (e.g., Jones \& Tetroe, 1987; Spelman Miller, 2000a; Spelman Miller et al., 2008; Wengelin, 2006; Wengelin et al., 2009; Xu \& Ding, 2014), a pause

Table 1 Characteristics of the student participants in the case study

\begin{tabular}{lllll}
\hline Number & Gender & Major & Writing test score & Group \\
\hline S1 & M & primary education & 14 & more-skilled \\
S9 & F & public administration & 8 & less-skilled \\
S10 & M & horticulture & 12 & more-skilled \\
S11 & F & tourism management & 14 & more-skilled \\
S12 & F & business administration & 7 & less-skilled \\
S14 & F & engineering management & 8 & less-skilled \\
S15 & F & broadcast journalism & 6 & less-skilled \\
S18 & F & primary education & 12.5 & more-skilled \\
\hline
\end{tabular}


threshold of 2 sec was adopted, thus any nonscribal period lasting for 2 sec or above would be identified as a pause. For each author, the number and the lengths of pauses made during the writing task were calculated to estimate the frequency and the duration of pausing behaviour of the participant. Pauses were further specified by their location in relation to the unfolding discourse, i.e., at the word, the sentence, and the paragraph level (Wengelin et al., 2009). SPSS 22.0 was used to generate the descriptive statistics.

The think-aloud protocols were transcribed following a set of rules (Appendix 2) adapted from Green (1998). The log file data of the eight targeted participants were reviewed, with all pauses exceeding the two-second threshold identified and annotated. Then open coded analysis was conducted with the qualitative data using ATLAS.ti 8.0. To ensure the validity of the coding practice, the first author invited a research assistant, who was a doctoral student in applied linguistics with similar research interest and experience of qualitative analysis, to independently code $30 \%$ of the transcriptions, which were randomly selected from the collection of the transcribed data. The two coders initially agreed on $83 \%$ of the coding results and then discussed and solved the disagreements before revising and finalizing the coding scheme, which was structured on the basis of a tripartite classification of pauses. This classification was built around the reoccurring keyword "problem" that emerged in the analysis of participants' selfreport data in the stimulated recall interviews and the post-task questionnaires. Participants tended to define a "problem" as any felt or perceived discrepancy between what the author saw in the produced text or visualized for the upcoming text and what $\mathrm{s} / \mathrm{he}$ thought or believed to be the correct or desired way of expression and/or communication. Based on this understanding, we categorized the pausing behaviours into three subtypes: observational, evaluative, and diagnostic pauses. An observational pause was operationalized as a pause during which the author inspected or took note of a "problem" in writing. An evaluative pause was operationalized as a pause during which the author formed an idea of the writing against some perceived standard or expectation, hence assessing a potential "problem". A diagnostic pause was made when the author identified a "problem" in writing by examining its symptomatic features. This classification is illustrated with sample think-aloud excerpts (italicized content was originally in English) below. Excerpt 1 demonstrates an observational pause during which the author observed the produced text and noticed the "problem" of having produced repeated content. In Excerpt 2, the author made an evaluative pause to assess the appropriateness of the drafted title. The evaluation was made against her received understanding of a good title, i.e., a good title should be representative in content and formal in style. The author in Excerpt 3 read back the produced sentence and decided that the use of the plural noun "difficulties" in the structure "no matter how ..." was problematic. Such a diagnosis led her to change the word to its adjective form, hence a diagnostic pause with immediate revision.

Excerpt 1

S9: As an adult ... As an adult, we should learn to ... deal with problems. It seems that I have mentioned this earlier. (15s)

Excerpt 2

S18: The title of the essay should reflect its content. Then how about when we meet the ... oh, no, no, this is not good, it doesn't sound formal enough (5s) how about the 
attitudes towards difficulties? This should be fine. The proper attitudes towards difficulties.

Excerpt 3

S15: This does not look right. In my view, when the difficulties are showing up in our life we should be faced with the challenge rather than escape it and no matter how difficulties, no matter how ... (10s) Yes, difficult, difficult [substitute "difficulties" with "difficult"]

\section{Results and discussion}

\section{Pause frequency and duration}

On the whole, the temporal statistics did not reveal much difference between the more-skilled and the less-skilled writers (Table 2). For both groups, pause time took up about half of the total task time, with the proportion of pause time slightly lower in the more-skilled group (49.77\%) than in the less-skilled group (59.76\%). The logging data further suggested that the pauses made by the less-skilled group tended to be higher in frequency and shorter in duration than those made by the more-skilled group. This overall tendency agreed with previous findings (Nie \& Li, 2016; Xu, 2011) that moreskilled writers paused less yet longer than their less-skilled peers.

This overall finding was in line with the general observation that the pausing patterns do not differ greatly among writers at different skill levels (Barkaoui, 2019; Schumacher et al., 1984). Nevertheless, empirical evidence is available that suggests writers would adopt different strategies during stages of the writing process based on their level of writing proficiency. For instance, Sasaki (2000) found experienced L2 writers spent more pausing time in pre-writing planning, whereas novice writers stopped at the boundary of each produced chunk. Xu and Ding (2014) revealed a similar pause time allocation pattern in that skilled L2 writers paused slightly less frequently in general yet significantly longer before they started to write. Comparable findings were also reported in $\mathrm{Xu}$ and Qi (2017), where the researchers found that the interval pausing patterns rather than the global pausing patterns were subject to the effect of writing skill. These findings suggest that skilled writers tend to employ more strategic pauses than lessskilled writers, whose pausing behaviours appear in a relatively random, passive manner. In the current study, a short preparation session was separated from the writing activity. Since all participants were allowed to draft their ideas within a designated period of time, the pre-writing activity of planning became more of an instructed rather than a self-initiated activity. Thus, more distinctive differences in the pausing

Table 2 Frequency and duration of pauses made by more- and less-skilled EFL writers

\begin{tabular}{lll}
\hline & $\begin{array}{l}\text { More-skilled writers } \\
(\boldsymbol{N}=\mathbf{4})\end{array}$ & $\begin{array}{l}\text { Less-skilled writers } \\
\mathbf{( N = 4 )}\end{array}$ \\
\hline Total task time (minutes) & 33.196 .05 & Mean SD \\
\hline Total pause time (minutes) & 16.526 .21 & 34.374 .01 \\
Total number of pauses (times) & 122.0023 .71 & 20.545 .77 \\
Mean pause duration (seconds) & 8.102 .30 & 156.2513 .15 \\
\hline
\end{tabular}


behaviours of the two groups might be brought to the fore in a writing task where no preparation stage was specified.

More refined analyses identified differences in the location of pauses made by the two groups of writers. In general, participants paused the most frequently at the word level and the least frequently at the paragraph level, with sentence-level pauses in between. This finding indicated that during the pause, the writer's attention was more often directed to fix a "local" problem than a "global" one (Heuring, 1984). Compared with the higher-level cognitive processes of organizing and planning, accessing lexical items is at the lower end of the writing process (Medimorec \& Risko, 2017). Participants' lopsided attention to lexical units of the discourse can be associated with deficits in vocabulary. As Witte and Faigley noted, the less competent writers "do not have working vocabularies capable of extending, in ways prerequisite for good writing, the concepts and ideas they introduce in their essays" (1981, p. 198). Crippled by their insufficient working vocabulary, the EFL writers could have got stuck at any lexical level of production. Participants' intensive pauses at the word level further suggested that both groups of writers were still at the novice level despite their test-proven differences in writing proficiency. This secondary finding endorsed a relativist view of the divide between skilled and unskilled writers, i.e., the boundary remains fluid and could be subject to contextual influences (Raimes, 1985) (Table 3).

A closer look at the word-level pauses revealed subtle clues to differentiate the two skill groups. While both groups made the greatest number of pauses between words, the less-skilled writers paused more than their more-skilled counterparts at all lexical levels, i.e., within (2.67:1), before (1.38:1), after (2.03:1), and between (1.53:1) words. Most noticeably, the less-skilled writers, on average, made more than 2.5 times the number of pauses within words made by the more-skilled writers. As within-word pauses can be taken as indicative of spelling difficulties, the less-skilled writers were likely to have trouble with the spelling of words that they memorized only vaguely. Though dictionary use was allowed in performing the writing task, consulting the dictionary took time, which was limited in the timed task and had thus been adopted as the last resort. In this sense, the less-skilled writers' pausing behaviour can be seen as an intuitive response to uncertainties that arise from their struggles in spelling a word

Table 3 Word-level pauses made by more- and less-skilled EFL writers

\begin{tabular}{lll}
\hline & $\begin{array}{l}\text { More-skilled writers } \\
(\boldsymbol{N = 4 )} \\
\text { Mean SD }\end{array}$ & $\begin{array}{l}\text { Less-skilled writers } \\
(\boldsymbol{N = 4 )}\end{array}$ \\
\hline Number of pauses within words & 13.752 .36 & 36.759 .74 \\
Mean pause duration within words & 7.122 .63 & 8.244 .23 \\
Number of pauses before words & 39.2513 .00 & 54.008 .21 \\
Mean pause duration before words & 7.403 .05 & 8.272 .24 \\
Number of pauses after words & 8.501 .91 & 17.2510 .05 \\
Mean pause duration after words & 7.341 .10 & 5.521 .71 \\
Number of pauses between words & 64.0012 .75 & 97.7516 .88 \\
Mean pause duration between words & 7.473 .64 & 8.012 .23
\end{tabular}

Note: According to the Inputlog manual, a "within words" pause is defined as "each pause within a word"; a "before words" pause is defined as "each pause before a word"; an "after words" pause is defined as "each pause after a word"; a "between words" pause is calculated as "a consecutive combination of a pause labelled 'after words' and 'before words"' (Leijten \& Van Waes, 2019, pp. 83-84). The mean pause duration data in the table are presented in units of seconds 
or in searching for the right word to start, to continue, or to end a chunk of ideas (cf. Raimes, 1985). With regard to the length of pauses at the lexical units, while the lessskilled group tended to have longer within-word, before-word, and between-word pauses, they did not pause as long after words as the more-skilled group did. One possible reason for this inconsistent behaviour was that the more-skilled group would habitually spend a little more time checking the word after it was produced.

Another interesting observation was the amount of time the less-skilled writers spent within the span of individual words. It is generally held that within-word pauses tend to last shorter than between-word pauses, as writing pauses tend to last longer at a higher text unit level, e.g., sentence-level pauses are longer than word-level pauses (Spelman Miller, 2000a; Wengelin, 2006). The average within-word pause duration for the lessskilled writers seemed to violate this general trend as it nearly tied before-word pause length and exceeded the duration of after-word and between-word pauses. This somewhat unexpected finding could provide further evidence for the students' lack of vocabulary knowledge as they may have to exert greater cognitive efforts to spell the words than to put thoughts into words. In comparison with the "local" concerns (cf. Heuring, 1984; Yang, 2010), which often deal with grammatical errors, one of the primary concerns of the less-skilled EFL writers in the study appeared to be "extra-local" and could have drained a fair amount of their limited attentional resources (Xu, 2018) (Table 4).

A similar tendency could be identified in the sentence-level pausing behaviours among the more-skilled and the less-skilled writers. On average, the less-skilled group paused more often and longer than the more-skilled group, a tendency consistently observed at various pause locations in relation to the sentence boundary. This result partially aligned with what was reported in previous literature on L2 writing pauses across proficiency levels (e.g., Nie \& Li, 2016; Xu, 2011, 2017). Révész et al. (2017) indicated that pauses at the sentence level were likely associated with monitoring pauses; the EFL writers were expected to display self-monitoring behaviour during these pauses. Furthermore, as L2 writers' recursive behaviours largely occurred at the sentence level (Raimes, 1985), during these pauses, the students would possibly go back to check their newly produced sentences and edit them if deemed necessary. This assumption was later confirmed by the students' retrospective data (see Section "Pausing behaviour and self-monitoring" below).

By contrast, the paragraph-level pauses were found to be the rarest among the three pause locations. The less-skilled writers generally made more such pauses and paused

Table 4 Sentence-level pauses made by more- and less-skilled EFL writers

\begin{tabular}{lll}
\hline & $\begin{array}{l}\text { More-skilled writers } \\
(\mathbf{N = 4 )} \\
\text { Mean SD }\end{array}$ & $\begin{array}{l}\text { Less-skilled writers } \\
(\mathbf{N}=\mathbf{4})\end{array}$ \\
\hline Number of pauses before sentences & 2.000 .82 & $\mathbf{M e a n}$ SD \\
Mean pause duration before sentences & 7.591 .49 .87 \\
Number of pauses after sentences & 2.001 .63 & 8.584 .36 \\
Mean pause duration after sentences & 5.234 .75 & 4.001 .41 \\
Number of pauses between sentences & 5.003 .46 & 5.401 .42 \\
Mean pause duration between sentences & 8.324 .02 & 6.256 .65 \\
\hline
\end{tabular}

Note: According to the Inputlog manual, a "before sentences" pause is defined as "each pause before a sentence"; an "after sentences" pause is defined as "each pause after a sentence"; a "between sentences" pause is calculated as "a consecutive combination of a pause labelled 'after sentences' and 'before sentences'" (Leijten \& Van Waes, 2019, pp. 8384). The mean pause duration data in the table are presented in units of seconds 
longer than the more-skilled writers, whose writing seemed to flow across paragraph boundaries. This finding was largely consistent with previous observations that proficient writers paused more often at higher text units where global planning activities were involved (Dennett, 1985; Moragne e Silva, M., 1988). Due to their limited L2 knowledge and experience, the less-skilled writers tended to pause predominantly at lower text units to mend problems that were "basic", i.e., essential to the linguistic encoding. In comparison, the more-skilled writers who were relatively free from such concerns would choose to make strategic pauses to gain overall control of their writing (Table 5).

As Medimorec and Risko (2017) suggested, the location of pauses matters prominently in modulating the writing process. Their study established a correlation between specifically located pauses and their related textual features in the immediate discourse. Likewise, the findings in the current study suggested that where the writers paused in their composition communicated important messages about their writing habits and the level of writing proficiency they had probably achieved.

\section{Pause types}

Drawing from the participants' retrospective comments, the profiled pauses were further divided into observational, evaluative, and diagnostic types (Table 6). Within each type, a more refined distinction was made by specifying the focus of the writer's redirected attention. For example, a "language" subtype within observational pause identified a pause during which the writer observed a potential problem concerning linguistic encoding, e.g., a misspelt word, inappropriate wording, a syntactic solecism.

As illustrated in Table 6, the less-skilled writers paused more frequently than the more-skilled writers in general. With regard to the focus of the writers' redirected attention, the less-skilled writers tended to pause to observe problems in content and language, to evaluate language-related issues and to diagnose linguistic errors. The moreskilled writers had a shared focus on linguistic features in observational and evaluative pauses while surpassed the less-skilled writers only in evaluative pauses out of contentrelated concerns. These three major pausing behaviours were further specified with the problems and concerns identified in each pause subtype (Table 7).

In accordance with the location-based findings, the pause subtypes converged around language-related aspects of the unfolding text. This was evidenced in the diversity and complexity of linguistically-driven pauses for observation, evaluation, and diagnosis. Apart from

Table 5 Paragraph-level pauses made by more- and less-skilled EFL writers

\begin{tabular}{lll}
\hline & $\begin{array}{l}\text { More-skilled writers } \\
(\mathbf{N = 4 )} \\
\text { Mean SD }\end{array}$ & $\begin{array}{l}\text { Less-skilled writers } \\
(\mathbf{N = 4 )} \\
\text { Mean SD }\end{array}$ \\
\hline Number of pauses before paragraphs & 1.251 .50 & 1.251 .26 \\
Mean pause duration before paragraphs & 3.103 .87 & 3.553 .39 \\
Number of pauses after paragraphs & 0.000 .00 & 0.250 .50 \\
Mean pause duration after paragraphs & 0.000 .00 & 0.581 .16 \\
Number of pauses between paragraphs & 2.502 .89 & 3.252 .63 \\
Mean pause duration between paragraphs & 2.943 .55 & 3.831 .98
\end{tabular}

Note: The mean pause duration data in the table are presented in units of seconds 
Table 6 Types of pauses made by more- and less-skilled EFL writers

\begin{tabular}{lll}
\hline Pause type & $\begin{array}{l}\text { More-skilled writers } \\
(\boldsymbol{N}=\mathbf{4})\end{array}$ & $\begin{array}{l}\text { Less-skilled writers } \\
(\boldsymbol{N}=\mathbf{4})\end{array}$ \\
\hline Observational pause & 0 & 0 \\
Structure & 2 & 5 \\
Content & 8 & 20 \\
Language & 0 & 0 \\
Format & 10 & 25 \\
Total & & \\
Evaluative pause & 1 & 1 \\
Structure & 2 & 0 \\
Content & 14 & 23 \\
Language & 0 & 0 \\
Format & 17 & 24 \\
Total & 0 & 0 \\
Diagnostic pause & 0 & 0 \\
Structure & 0 & 6 \\
Content & 0 & 0 \\
Language & 0 & 6 \\
Format & 0 & 0 \\
Total & 0 & \\
Grand total & 0 & \\
\hline
\end{tabular}

this overall tendency, subtle differences were detected in pausing behaviours between writers at different skill levels. In content-related observational pauses, while the less-skilled writers worried about falling short of the expected word count, the more-skilled writers noticed missing content. In language-related observational pauses, the less-skilled writers most frequently found themselves troubled or frustrated by their inability to convey the intended message or failure to convey it appropriately. The more-skilled writers, however, would more likely notice problems that could influence the quality of the writing (e.g., repetitive wording). In addition, as their skills improved, writers seemed to get over technical problems such as spelling and capitalization, which still sat on the minds of some less-skilled writers.

Both groups made few evaluative pauses to assess the structure and content of their writing, with only one instance for each group on structural appropriateness. While some more-skilled writers paused to assess the title and the content of the writing, this evaluative activity was missing among the less-skilled writers who may have devoted their attention to mending the language problems, as indicated by the bunch of problems they had specified. Such differences were more pronounced in the diagnostic pause data, which only registered language-related problems identified by the less-skilled writers. Since evaluative and diagnostic pauses would often lead to revising or editing, this finding suggested that the less-skilled writers could have spent a substantial amount of time and effort going over the words and sentences just to make them grammatically correct.

A comparative look at the think-aloud protocols revealed more detailed differences between the two groups in terms of their pausing preferences. Both groups exercised self-observation, self-evaluation, and self-monitoring to tackle problems concerning the structure, content, and language of their writing, yet the way in which these cognitive 
Table 7 Subtypes of pauses made by more- and less-skilled EFL writers

\begin{tabular}{|c|c|c|}
\hline Pause subtype & $\begin{array}{l}\text { More-skilled writers } \\
(N=4)\end{array}$ & $\begin{array}{l}\text { Less-skilled writers } \\
(N=4)\end{array}$ \\
\hline \multicolumn{3}{|l|}{ Observe a content-related problem } \\
\hline Missing content & 2 & 1 \\
\hline Repeated content & 0 & 1 \\
\hline Insufficient word-count & 0 & 3 \\
\hline Total & 2 & 5 \\
\hline \multicolumn{3}{|l|}{ Observe a language-related problem } \\
\hline "Don't know how to put it" & 2 & 6 \\
\hline Inappropriate expression & 0 & 4 \\
\hline Capitalization & 0 & 1 \\
\hline Spelling & 0 & 3 \\
\hline Incorrect wording & 0 & 2 \\
\hline Repetitive wording & 5 & 3 \\
\hline Grammatical error & 1 & 1 \\
\hline Total & 8 & 20 \\
\hline \multicolumn{3}{|c|}{ Evaluate a structure-related aspect of the written text } \\
\hline Structural appropriateness & 1 & 1 \\
\hline Total & 1 & 1 \\
\hline \multicolumn{3}{|c|}{ Evaluate a content-related aspect of the written text } \\
\hline Title appropriateness & 1 & 0 \\
\hline Content richness & 1 & 0 \\
\hline Total & 2 & 0 \\
\hline \multicolumn{3}{|c|}{ Evaluate a language-related aspect of the written text } \\
\hline Expression conciseness & 0 & 2 \\
\hline Expression appropriateness & 1 & 4 \\
\hline Part of speech correctness & 2 & 2 \\
\hline Verb usage correctness & 0 & 4 \\
\hline Syntactic correctness & 1 & 0 \\
\hline Sentence structural complexity & 1 & 0 \\
\hline Sentence structural appropriateness & 2 & 2 \\
\hline Nominal countability & 2 & 1 \\
\hline Spelling correctness & 1 & 3 \\
\hline Wording appropriateness & 4 & 4 \\
\hline Wording correctness & 0 & 1 \\
\hline Total & 14 & 23 \\
\hline \multicolumn{3}{|l|}{ Diagnose a language-related problem } \\
\hline Wordy expression & 0 & 1 \\
\hline Part of speech misuse & 0 & 1 \\
\hline Spelling mistake & 0 & 4 \\
\hline Total & 0 & 6 \\
\hline
\end{tabular}

processes were manifested in their pausing behaviours and their responses to the detected problems differed to varying degrees. For instance, after assessing the appropriateness of the structure of their writings, the less-skill writer separated the exiting 
paragraph (Except 4), whereas the more-skilled writer chose to rearrange the sentence order (Excerpt 5). In response to content-related problems, both the more-skilled and the less-skilled writers addressed insufficiency by adding linguistic units on the lexical, phrasal, clausal, or sentential level (Excerpts 6-10). In addition, the less-skilled writers also attended to surface or technical issues such as repetition (Excerpt 8) and word count (Excerpt 9), which was not found among the more-skilled writers. In contrast, while some more-skilled writers assessed whether the essay was fully developed and added sentences to enrich her writing (Excerpt 10), no such evaluative activities were reported by the less-skilled writers.

Excerpt 4

S14: Should I put these ideas in two separate paragraphs? (5s) [review the preceding text] Therefore, Therefore, there are a variety of difficulties in our lives. I shall divide this whole chunk here into two paragraphs. [Move "Some of us are ..." off the preceding text to form a separate paragraph.]

\section{Excerpt 5}

S11: There seems to be a better place for this sentence later in the text. Better to put it in Paragraph Three. (8s) [move "to live a life is like claiming a steep mountain, despite the tiredness during the whole journey, the significant view you eventually receive would overcome all the negative emotion" in p1 to p3; substitute "to" with "To"]

\section{Excerpt 6}

S18: When we meet difficulties ... er, Paragraph Two is still missing an opener. (5s) When we meet difficulties, some people ... [add "When we meet with difficulties," to the beginning of $\mathrm{p} 2]$

\section{Excerpt 7}

S9: I seem to have missed one sentence ... about the reason, $\mathrm{mm}$... (20s) Find ... find the cause and take it as ... [add "Find the cause and" to p2s3]

\section{Excerpt 8}

S9: As an adult ... As an adult, we should learn to ... solve problems. I seem to have mentioned this before. (15s)

\section{Excerpt 9}

S12: Mm ... not yet reached the target word count. Got to write about something else. (5s)

Excerpt 10

S18: No matter how difficult the learning process is, this may not be enough (5s) [review p3] Obviously there are difficulties in her life, but she has always faced life with a positive attitude, and she has always kept on learning, something seems to be missing here $(5 \mathrm{~s})$ She keeps on learning like a normal student, no matter how difficult the learning process is. What about her life? Life should be more difficult. Yeah, I should talk about her blindness first. [add "She never loses heart to the life" to p3s3] Even, even, and she even [add "even"] persisted in studying like other normal students however tough her studying process is. So, in the end she won, through continuous efforts (5s) By continuous efforts, she finally achieves success like a normal person, without falling her own expectations (5s) she achieved success at last.

With regard to the language-focused pauses, both groups had instances during which they observed, evaluated, or diagnosed language-related problems 
and made responses accordingly (Excerpts 11-18). For instance, when they had difficulty putting their intended meaning in words, the less-skilled writers may get around the problem by dropping the idea or choosing an alternative sentence pattern that would save the trouble of mentioning the unfamiliar word or phrase (Excerpt 11). The more-skilled writers, however, may resort to paraphrasing or other interpretative means to try to convey the intended message (Excerpt 12). Apart from the difficulty in expressing themselves, the lessskilled writers also found themselves confronted with the task of addressing grammatical and spelling issues, which were normally not the concern of the more-skilled writers. However, not all these language-targeted pauses would lead to conducive changes to the writing. In some cases, the problem could remain unaddressed, or a new problem could be introduced because of a false evaluation or diagnosis; in others, a proper evaluation or diagnosis may not lead to a proper revision, possibly due to the author's limited or erroneous knowledge (Excerpt 13). In this regard, the more-skilled writers seemed to have adequate knowledge to assist their judgement and revision of a spotted language problem (Excerpts 15 and 16). Apart from basic concerns about correctness and appropriateness, they may also pay additional attention to the more "advanced" aspects of their language production, such as structural balance and syntactic sophistication (Excerpts 17 and 18).

Excerpt 11

S12: Mm, these difficulties go through our life, these difficulties ... go through our life, go through? Don't know how to put it (5s) Mm ... our life is full of difficulties, be filled with. Our life is filled with ... [substitute "there are" with "our life is filled with"]

Excerpt 12

S18: Mm ... real gold fears no fire, is there any other proverb about difficulties? Mm, in the test of difficulties, mm ... (10s) Put it in another way, because people can learn lessons from difficulties, because people can learn some lessons from ...

\section{Excerpt 13}

S12: But it still has possibility that we can't success, should the verb form here be success or succeed? (10s) success, it still has possibility that we can't success.

\section{Excerpt 14}

S18: Nobody can become successful easily without ... Shall I use overcome or meet? Nobody can succeed easily, because they fail to overcome the difficulties they meet. $\mathrm{Mm}$, it should be without overcoming difficulties (10s)

\section{Excerpt 15}

S11: To live a life, living a life, better to use an -ing verb as the subject (10s) ... can be analogized to climb a mountain, to climb a steep mountain [substitute "To live a life" with "Living a life"]

\section{Excerpt 16}

S18: Besides, difficulties are also an opportunity because people can learn a lesson from which they go through and ... This is also an opportunity, because people can learn from it, and ... should I use a different sentence pattern here? (10s) [review the sentence again] Difficulty is an opportunity, people can learn from it ... and by, only by perhaps can't be used here. Then I'll use and by, and by reflecting ... their behaviours, their behaviours in ... dealing with difficulties, dealing with the problems, they can see the ... 


\section{Excerpt 17}

S18: [review the opening of $\mathrm{p} 2$ ] When we meet with difficulties, some people choose to be faced with them bravely but others will feel timid so they give up their tasks or dreams halfway ... which, perhaps would lead to a pity at last, which may lead to their ... no success, that is failure (10s) failure, and, achieve nothing in life, may lead to their failure and achieve nothing. Can I combine these two? (5s) may lead to their failure and make them achieve nothing.

\section{Excerpt 18}

S18: but others ... showcase a state of being timid, but if I only use the adjective timid, this sentence may look too simple (5s) some people choose to be faced with them bravely but others feel timid.

\section{Pausing behaviour and self-monitoring}

These pausing behaviours conveyed significant messages about the underlying cognitive process of the EFL learners' real-time writing. Overall, they revealed patterns of self-monitoring as the writer's attention was redirected to address problems that emerged in the writing process. In general, the more-skilled writers demonstrated stronger self-monitoring awareness and skills by making pauses targeting the comparatively higher-level features of the text and afterwards making revisions to address the detected problems. The less-skilled writers made revisions mainly to address lexical and syntactic problems spotted in the text. Their observational and evaluative pauses may not lead to revisions or may result in erroneous revisions due to improper judgement or inadequate knowledge. This discrepancy seemed to suggest that though the lower-skilled writers had showcased self-monitoring awareness in making the pauses, they may still lack the ability to exercise self-monitoring in real practice.

In terms of the way how self-monitoring was exercised in performing the online writing task, the two groups featured different monitoring patterns. The more-skilled writers adopted real-time self-monitoring throughout the formulation, execution, and monitoring processes of writing (Kellogg, 1996) by revising immediately after identifying a problem in the produced or the unfolding text. Drawing from the pausing and revising behaviours they demonstrated, their monitoring patterns could be roughly summarized as "selfobservation $\rightarrow$ self-correction" or "self-evaluation $\rightarrow$ self-correction". In comparison, the less-skilled writers mainly exercised self-monitoring during the production of the text, and assumed various monitoring patterns including "real-time self-revision", "self-observation", "self-evaluation", "self-observation $\rightarrow$ self-correction", "self-evaluation $\rightarrow$ self-correction", "self-observation $\rightarrow$ self-diagnosis $\rightarrow$ self-correction", "self-observation $\rightarrow$ self-evaluation $\rightarrow$ selfcorrection" or "self-observation $\rightarrow$ self-diagnosis $\rightarrow$ self-correction". This more heterogenous self-monitoring pattern suggested that the less-skilled writers may not revise after observation or evaluation of a potential problem. Hence their self-monitoring practice may not lead to conducive effects on their writing performance. Moreover, in cases where their observation and evaluation lead to revisions, they may need the mediation of more procedural 
activities, hence a need for additional pauses to assist the process, which could result in more frequent, less organized pausing behaviours.

A qualitative analysis of the students' retrospective comments from the interviews and the questionnaires further revealed the possible reasons for the differences in selfmonitoring patterns between writers of different skill levels. These differences could be sorted into three themes, i.e., self-monitoring awareness, writing knowledge and experience, and writing habit.

First, the features of the writing pauses made by writers of different skill levels indicated differences in self-monitoring awareness. As the post-task questionnaire data indicated, both the more- and the less-skilled writers were aware of problem-finding and error-detecting and would "keep an eye" on the potential mistakes in writing. Those who were more skilled tended to focus on more "advanced" problems (e.g., the logic of content, coherence of writing, word choice) and would make timely corrections after spotting a problem. By contrast, their less-skilled peers mainly spent their pauses checking for spelling errors and other mechanical errors in lexicon and grammar. Similar to the more-skilled writers, they would also exercise possible corrections after noticing the problematic expression. However, some lowachievers confessed that they at times felt unable to make a proper judgement on the correctness or appropriateness of their language, and hence incapable of making corrections. For example, S12 reported in the questionnaire that she "felt something was wrong", yet she "was not sure what was wrong" or "how to make it right". S9 explained that she would make corrections despite her uncertainty about the "strange" language she produced, yet she seriously doubted whether her correction "would make things better". S15 mentioned that she was able to spot grammatical mistakes that were "simple", yet often found herself clueless when trying to correct grammatical mistakes that were "relatively complicated".

Second, the writing pause differences between writers of different levels could also be attributed to differences in writing knowledge and experience. The questionnaire and the interview data showed that none of the moreskilled writers found the writing task difficult and that they all agreed that the topic was straightforward and easy to write about. The two skilled writers, S1 and S11, who shared their learning experience in the interview, owed their fluency much to the words and phrases they had "accumulated", which allowed them to make changes in their writing "without thinking too much". On the contrary, it was no surprise that the less-skilled writers generally found the topic difficult to write about. They were often struggling against the linguistic encoding pressure from various sources, e.g., word spelling, vocabulary, sentence structure, and grammar. Therefore, they would often pause to check whether the language was appropriate, which could explain the prominent proportion of observational and evaluative pauses in their writing practice.

The third theme emerged in the students' retrospective comments might be associated with writing habit, as the participants reported different writing and revising habits in their questionnaire responses. Among them, the two more-skilled writers (S1 and S11) and the two less-skilled writers (S9 and S15) reported a mixture of revising habits: some revisions happen during 
writing while others after writing. For these writers, the self-monitoring activities took place mainly in the subprocesses of production, reviewing and revising. There were more refined differences in this shared habit, however, as writers of different skill levels would likely revise for different purposes. In the recall interview with $\mathrm{S} 1$, the more-skilled writer mentioned that he would revise while writing mostly to correct spelling mistakes and improve wording, while the main focus of his post-writing revision was on the overall logic of the text, with mainly polishing efforts such as rearranging the sentence order to make the writing more coherent. Another more-skilled writer, S11, described in her interview that she would often go back to read what she had written to check for appropriateness and logical consistency so that she could revise promptly. This rereading practice was noticed earlier by Raimes (1985) as she found her ESL students would "frequently read back over phrases and sentences just written" while the two students with high language proficiency "did not continually go back to read and repeat what they had just written" (Raimes, 1985, p. 242). The rereading habit, as confessed by S11, seemed to identify herself as a less proficient learner, yet this might also be thought of as a habitual behaviour that assisted learners with limited L2 proficiency to monitor and gain control of their writing.

\section{Limitations and implications}

The findings of the current study need to be considered with several limitations. One principal limitation is the small number of students involved in the study. There was a practical challenge in recruiting suitable and representative subjects who were invited to partake in a series of tasks and surveys. Therefore, the number of participants was small, and the preliminary findings on the EFL learners' pause profiles should be read as based on multiple cases that provide evidence for writing pause behaviours of individual writers. Nevertheless, this preliminary attempt allowed us to test the feasibility of a demanding study design that draws from a range of data sources. Following a bottom-up approach, more generalizable findings can be obtained with larger samples.

Another methodological limitation is the use of topic sentences as a content guide for the students, who were likely to have been substantially facilitated by the given statements in planning their writing. This specific design could have influenced the results of the students' pausing behaviours at the paragraph level, since they had already been shown a model structure of the essay and may not bother to make extra efforts to come up with alternative structures. The underlying consideration for this specific design, however, was to reduce the inhibiting effect of content on L2 writer's performance (Myles, 2002, p.4). As it was found in the pilot study that the target students would likely suffer from such an influence due to limited L2 proficiency, the authors decided to reduce task complexity by providing content support. This consideration was intended to help the students conceptualize the content of their essay so that they could focus more effectively on linguistic encoding, with which most of them were still struggling. Previous studies (Kormos, 2011; Révész et al., 2017) have connected task complexity with linguistic complexity indices, and it is hypothetically reasonable to 
assume that task complexity also influences pauses as content support was found to lead to less pausing (Révész et al., 2017). Nonetheless, it remains to be empirically testified as to how specific pausing behaviours are subject to task complexity.

With regard to task complexity, the findings on the paragraph-level pauses in the current study should also be considered by taking into account the limited length of the essays. An alternative, more likely explanation for the overall paucity of paragraph-level pauses was that participants were not expected to produce a full essay but a few sentences arranged in a logical order. In terms of text length, the recommended 150 words for participants' written production could make an independent paragraph in its own right. To put this into perspective, the recommended paragraph length is around 75-150 words for scientific writing that features brevity and conciseness (Bahadoran et al., 2018). Therefore, unless very short paragraphs were intended, which would possibly result in a fragmented piece of writing, there was no obvious practical need for the writer to separate his/her composition into multiple paragraphs, which was evidenced in several one-paragraph samples produced by the EFL writers. Therefore, if participants were requested to produce an extended-length text (e.g., a full paper) in a writing pause study, the results may be somewhat different and more revealing of pausing behaviours at a high text unit level.

Despite these limitations, this study has generated some interesting observations that may inform theoretical understanding of writing pauses and their characteristics and suggest possible ways to make productive use of pauses to aid writing. In particular, the "problem"-oriented taxonomy of writing pauses proposed in the study takes an "insider" perspective by reconceptualizing pauses as part of writers' locally contingent response to the demand for the global control of the unfolding text. By tapping into the composing as well as the thinking processes of individual writers, we would be in a better position to explain and appreciate the complex and idiosyncratic process of writing and would be able to help others understand the enigma of writing better.

As researchers in the field of writing studies, we have noticed a tendency for empirical works to focus on specific sub-processes of writing (e.g., execution, pause, revision). This development is motivated and underpinned by advances in technology, which have enabled a closer and finer-grained analysis of the behavioural measures and features of the written product. Focused analyses as that conducted in the current study would continue to offer glimpses into the less well-understood aspects of writing as a complex cognitive activity. The pausing patterns revealed by this case study point to the idiosyncrasies of L2 writing pauses, since a mixture of factors may influence pausing behaviours, including language background (Jones \& Tetroe, 1987), study experience (Spelman Miller et al., 2008), writing mode (Van Waes \& Schellens, 2003) and transcription fluency (Alves et al., 2008; Medimorec \& Risko, 2017; Olive et al., 2009; Van Waes et al., 2019). Further descriptions of L2 writer's situated writing practice are called for to yield a better understanding of the intricate interaction between factors that conflate to influence the writing process and product. 
As EFL teachers who have long been teaching tertiary-level writing courses, we feel increasingly pressured to introduce new ideas and methods to the class. This pressure comes largely from the drastic changes in the way students write and the way they approach their writing. Quite noticeably, it used to be commonplace to see college students in Chinese universities taking handwritten notes; yet this has become an increasingly rare sight, especially in schools located in developed regions where students' access to digital gadgets is extensive (Wang et al., 2011). Nowadays, students prefer to keep "e-notes" on their laptops, tablets, or smartphones, and the notes can be easily stored, accessed, and edited. Many are using note-taking APPs that offer a rich assortment of aids to make writing interactive and fun. These and many other new trends in students' writing practices call for critical reflections on longstanding understandings of writing and serious research efforts to examine and assess such emerging trends. Recent studies such as Vandermeulen et al. (2020) shed inspiring light on the value of reaping educational benefits from quantified and visualized reports. Since pausing can reveal much about the complex cognitive underpinnings of writing and the writer's cognitive styles and metacognitive activities, students' pause profiles can be fruitfully used to inform the writing classroom and to make it more effective and engaging for learners at different levels.

\section{Conclusion}

This study explored the real-time computer-aided writing practices of EFL learners by profiling their pausing behaviours and their cognitive and physical activities conducted in the pausing episodes. The pauses were located across the boundaries of text units and were sorted based on the acts the pauses intended to perform in the writing task. In an effort to reconceptualize pauses as strategic problem-solving responses, we specified three subtypes of pauses, including observational, evaluative, and diagnostic pauses. By applying this conceptual model to examine eight cases of EFL writers, the study identified pausing tendencies among learners with different levels of writing skills. A mixture of quantitative and qualitative measures was adopted to chart the real-time activities of individual writers. The study showed that the EFL writers' pausing behaviour featured attention and efforts directed to address language-related concerns in text production. While the more-skilled writers made additional pauses for observing and evaluating higher-level units of text, the less-skilled writers mainly struggled to address lower-level problems of word and grammar. These identified characteristics further revealed features and patterns of self-monitoring exercised by writers at different skill levels. Both groups of writers had showcased their awareness of self-monitoring their writing process, yet the more-skilled writers tended to have a stronger ability to exercise effective self-monitoring than their less-skilled counterparts, whose self-monitoring activities may not lead to meaningful effects on their performances. While the more-skilled writers demonstrated consistent patterns of self-correction guided by observation and evaluation, the lessskilled writers had a range of self-monitoring patterns, with some mediated by additional subprocesses that may result in more pausing activities.

Despite its limitations, this small-scale multi-method study offers a glimpse into how EFL learners would respond to a real-time online writing task by mobilizing and modulating knowledge and linguistic resources at their disposal. By seeking coordination between a mixture of measures and data, it presents additional empirical evidence to the literature on computer-aided writing examined by computer-aided tools. With enhanced measures and 
data sources, we may expect the advancing technological aids to open new avenues of research for a more thorough and convincing analysis of the cognitive processes in writing.

\section{Appendix 3}

\section{Students' writing samples}

S11 (a more-skilled writer).

To Live is to Fight

Life is saturated with all kinds of dilemmas. After all, it won't be called as "life" if you only have joy and comfort during this less-than-a-hundred-year trip.

Nevertheless, various individuals tackle with setbacks in their own ways due to the affluent amount of characteristics. Some brave people would face up to difficulties directly, while others, because of their timidity, they choose to withdraw and escape from it. From my perspective, however, comparing these two options, I incline to select the former attitude for the following reason.

It is my point of view that we need, and we should, fight against negative conditions with all the courage we have. Living a life can be analogized to climbing a steep mountain. Despite the tiredness during the whole journey, as long as you have a firm belief that "you can overcome it", you will reach the peak of the mountain and enjoy the significant view eventually. Similarly, one would attain unexpected satisfaction from the problem or the trouble he solves, provided he preserves an active attitude.

To draw to a brief conclusion, difficulties are unavoidable but solvable. Thus, instead of being a coward, we ought to stand out and be a fighter to beat them down.

S15 (a less-skilled writer).

Facing difficulties.

What is known to all is that the life is always fulled with difficulties. Just like math is very hard to you or you are faced with the pressure of entering a higher school and maybe you are facing worse thing than the above all. Nevertheless, somebody always escapes the challenge when she or he feel so hard and they will choose give up it. On the

\section{Appendix 1}

Table 8 Rating rubric for the timed online writing task (based on CET-4 writing rubric)

\begin{tabular}{|c|c|c|c|}
\hline Points & Organization & Content & Language \\
\hline 0 & No organization & Little idea & $\begin{array}{l}\text { Incorrect spellings or a few isolated } \\
\text { words }\end{array}$ \\
\hline 2 & No focus; disorganized & $\begin{array}{l}\text { Ideas random, } \\
\text { inappropriate or illogical }\end{array}$ & $\begin{array}{l}\text { Incomplete or incorrect sentences; severe } \\
\text { errors that affect meaning }\end{array}$ \\
\hline 5 & $\begin{array}{l}\text { Attempts to focus; minimal } \\
\text { organization }\end{array}$ & $\begin{array}{l}\text { Ideas mixed; few } \\
\text { transitions }\end{array}$ & $\begin{array}{l}\text { Monotonous errors that interfere with } \\
\text { meaning }\end{array}$ \\
\hline 8 & $\begin{array}{l}\text { Single focus; some lapses of } \\
\text { flaws in organization }\end{array}$ & $\begin{array}{l}\text { Ideas not well supported } \\
\text { or elaborated }\end{array}$ & $\begin{array}{l}\text { Little variety in syntax; some evident } \\
\text { errors }\end{array}$ \\
\hline 11 & $\begin{array}{l}\text { Single focus; logical } \\
\text { organization }\end{array}$ & $\begin{array}{l}\text { Ideas appropriate and } \\
\text { varied }\end{array}$ & Varied sentence structure; few errors \\
\hline 14 & $\begin{array}{l}\text { Single, distinct focus; logical } \\
\text { progression of ideas }\end{array}$ & $\begin{array}{l}\text { Details effective, vivid, } \\
\text { explicit and pertinent }\end{array}$ & Very few, if any, errors \\
\hline
\end{tabular}

Note: The rating practice adopts a holistic rating format. Scores are given based mainly on the overall impression the composition creates on the rater, rather than following the practice of deducting points based on the number of errors (e.g., grammatical solecisms) identified in the composition. A total of five levels are specified in this rating rubric, and minor deviations from the description for each level may result in the loss or gain of one point. The highest score (full score) is 15 , and the lowest score is zero 


\section{Appendix 2}

Table 9 Illustration of transcription rules

\begin{tabular}{ll}
\hline Symbol & Explanation \\
\hline$\ldots$ & Incoherent speech \\
& Ambiguous speech \\
& Phonetics during word spelling \\
() & Writing behaviour observed in Camtasia screen recording \\
$(5 \mathrm{~s}),(1 \mathrm{~m})$ & The part that the researcher observes but does not appear in the think-aloud protocols \\
$\mathrm{p}, \mathrm{s}$ & Abbreviations of paragraphs and sentences, e.g. p1s1 stands for the first sentence in Paragraph 1 \\
\hline
\end{tabular}

contrary, there are person will choose try to overcome the problem. As an old saying goes: "failer is the mother of success." In my view, when the difficulties are showing up in our life we should be faced with the challenge rather than escape it and no matter how difficult, only when we do it, can we no feel regrets in the end of our life. Therefore, we don't have to fear the failer and we should be faced with all the difficulties in our life and make great efforts to overcome it.

\section{Abbreviations}

EFL: English as a Foreign Language; ESL: English as a Second Language; L1: First Language; L2: Second Language

\section{Acknowledgements}

The authors would like to thank the anonymous reviewers for offering insightful comments and detailed revision suggestions on an earlier draft of the paper. Sincere gratitude also goes to all the student participants and the teacher raters for their contribution to this study.

\section{Authors' contributions}

CS and NC conceptualized and designed the study. CS conducted the study. CS and NC drafted the original manuscript. Both authors read and approved the final manuscript.

\section{Authors' information}

Cuiping Shen is an associate professor at the Faculty of Foreign Languages of Shanghai Open University. Her research interests include second language writing and computer-assisted language learning and teaching.

Ningyang Chen is a post-doctoral researcher at the School of Foreign Languages of Soochow University. Her research interests include writing in English as an Additional Language and academic biliteracy.

\section{Funding}

The authors received no funding for the research.

Availability of data and materials

The datasets analysed in the current study are available from the corresponding author on reasonable request.

\section{Declarations}

Competing interests

The authors declare no competing interests regarding the research, authorship, and/or publication of this article.

\section{Author details}

${ }^{1}$ Faculty of Foreign Languages, Shanghai Open University, Shanghai, People's Republic of China. ${ }^{2}$ School of Foreign Languages, Soochow University, Jiangsu, People's Republic of China.

Received: 20 April 2021 Accepted: 11 July 2021

Published online: 16 August 2021

\section{References}

Alves, R. A., Castro, S. L., \& Olive, T. (2008). Execution and pauses in writing narratives: Processing time, cognitive effort and typing skill. International Journal of Psychology, 43(6), 969-979. https://doi.org/10.1080/00207590701398951.

Baaijen, V. M., Galbraith, D., \& De Glopper, K. (2012). Keystroke analysis: Reflections on procedures and measures. Written Communication, 29(3), 246-277. https://doi.org/10.1177/0741088312451108.

Bahadoran, Z., Jeddi, S., Mirmiran, P., \& Ghasemi, A. (2018). The principles of biomedical scientific writing: Introduction International Journal of Endocrinology and Metabolism, 16(4), e84795. https://doi.org/10.5812/ijem.84795. 
Barkaoui, K. (2019). What can L2 writers' pausing behavior tell us about their L2 writing processes? Studies in Second Language Acquisition, 41(3), 529-554. https://doi.org/10.1017/S027226311900010X.

Barnhisel, G., Stoddard, E., \& Gorman, J. (2012). Incorporating process-based writing pedagogy into first-year learning communities: Strategies and outcomes. The Journal of General Education, 61(4), 461-487. https://doi.org/10.1353/jge.2012. 0041 .

Blyler, N. R. (1987). Process-based pedagogy in professional writing. International Journal of Business Communication, 24(1), 51-60. https://doi.org/10.1177/002194368702400113.

Chan, S. (2017). Using keystroke logging to understand writers' processes on a reading-into-writing test. Language Testing in Asia, 7(1), 1-27.

Chenu, F., Pellegrino, F., Jisa, H., \& Fayol, M. (2014). Interword and intraword pause threshold in the writing of texts by children and adolescents: A methodological approach. Frontiers in Psychology, 5, 182.

Chukharev-Khudilaynen, E. (2014). Pauses in spontaneous written communication: A keystroke logging study. Journal of Writing Research, 6(1), 61-84. https://doi.org/10.17239/jowr-2014.06.01.3.

Deane, P., Odendahl, N., Quinlan, T., Fowles, M., Welsh, C., \& Bivens-Tatum, J. (2008). Cognitive models of writing: Writing proficiency as a complex integrated skill. ETS research report series, i-36.

Dennett, J. (1985). Writing technical English: A comparison of the process of native English and native Japanese speakers. In Doctoral dissertation. University of Colorado at Boulder.

Flower, L. S., \& Hayes, J. R. (1977). Problem-solving strategies and the writing process. College English, 39(4), 449-461. https:// doi.org/10.2307/375768

Flower, L. S., \& Hayes, J. R. (1981). A cognitive process theory of writing. College Composition and Communication, 32(4), 365387. https://doi.org/10.2307/356600

Godwin-Jones, R. (2018). Second language writing online: An update. Lanquage Learning \& Technology, 22(1), 1-15.

Green, A. (1998). Verbal protocol analysis in language testing research: A handbook. Cambridge: Cambridge University Press.

Hayes, J. R., \& Flower, L. S. (1987). On the structure of the writing process. Topics in Language Disorders, 7(4), 19-30. https:// doi.org/10.1097/00011363-198709000-00004

Heuring, D. L. (1984). The revision strategies of skilled and unskilled ESL writers: Five case studies. Houston: Paper presented at the 18th annual TESOL convention.

Janssen, D., van Waes, L., \& van den Bergh, H. (1996). Effects of thinking aloud on writing processes. In C. M. Levy, \& S. Ransdell (Eds.), The science of writing: Theories, methods, individual differences, and applications, (pp. 233-250). Mahwah, NJ: Lawrence Erlbaum Associates.

Jones, S., \& Tetroe, J. (1987). Composing in a second language. In A. Matsuhashi (Ed.), Writing in real time, (pp. 34-57). Norwood, NJ: Ablex.

Kellogg, R. T. (1996). A model of working memory in writing. In C. M. Levy, \& S. Ransdell (Eds.), The science of writing: Theories, methods, individual differences and applications, (pp. 57-71). Mahwah, NJ: Erlbaum.

Kellogg, R. T. (1999). Components of working memory in text production. In M. Torrance, \& G. Jeffery (Eds.), The cognitive demands of writing: Processing capacity and working memory effects in text production, (pp. 43-61). Amsterdam: Amsterdam University Press.

Kellogg, R. T. (2001). Competition for working memory among writing processes. American Journal of Psychology, 114(2), 175191. https://doi.org/10.2307/1423513.

Kormos, J. (2011). Task complexity and linguistic and discourse features of narrative writing performance. Journal of Second Language Writing, 20(2), 148-161. https://doi.org/10.1016/j.jslw.2011.02.001.

Latif, M. M. A. (2008). A state-of-the-art review of the real-time computer-aided study of the writing process. International Journal of English Studies, 8(1), 29-50

Laver, J. (1995). Principles of phonetics. Cambridge: Cambridge University Press.

Leijten, M., \& Van Waes, L. (2012). Inputlog 4.0: Keystroke logging in writing research. Studies in Writing, 25, 363-366.

Leijten, M., \& Van Waes, L. (2013). Keystroke logging in writing research: Using Inputlog to analyze and visualize writing processes. Written Communication, 30(3), 358-392. https://doi.org/10.1177/0741088313491692.

Leiiten, M. \& Van Waes, L. (2019). Help documentation (in progress) draft 03 may 2019. https://www.inputlog.net/wp-content/ uploads/Inputlog_manual.pdf accessed 23 June, 2021.

Matsuhashi, A. (1981). Pausing and planning: The tempo of written discourse production. Research in the Teaching of English 15(2), 113-134

Medimorec, S., \& Risko, E. F. (2017). Pauses in written composition: On the importance of where writers pause. Reading and Writing, 30(6), 1267-1285. https://doi.org/10.1007/s11145-017-9723-7.

Moragne e Silva, M. (1988). A study of composing in a first and a second language. Texas Papers in Foreign Language Education, 1(2), 132-151.

Myles, J. (2002). Second language writing and research: The writing process and error analysis in student texts. Tesl-Ej, 6(2), 1-20.

Nie, Y., \& Li, Z. (2016). Jiyu jishu zhichi de yingyu xuexizhe xiezuo tingdun xingwei fenxi [a study on pause in English learners' writing process based on technology]. Xi'an Dianzi Keji Daxue Xuebao (Shehui Kexue Ban) [Journal of Xidian University (Social Science Edition)], 26(1), 93-101.

Olive, T., Alves, R. A., \& Castro, S. L. (2009). Cognitive processes in writing during pause and execution periods. European Journal of Cognitive Psychology, 21(5), 758-785. https://doi.org/10.1080/09541440802079850.

Pandey, A. (2012). Rethinking process-based writing approaches in the ESOL middle school classroom: Developing linguistic fluency via hybrid pedagogies. TESOL Journal, 3(4), 659-697. https://doi.org/10.1002/tesj.39.

Raimes, A. (1985). What unskilled ESL students do as they write: A classroom study of composing. TESOL Quarterly, 19(2), 229258. https://doi.org/10.2307/3586828.

Reither, J. A. (1985). Writing and knowing: Toward redefining the writing process. College English, 47(6), 620-628. https://doi. org/10.2307/377164

Révész, A., Kourtali, N.-E., \& Mazgutova, D. (2017). Effects of task complexity on L2 writing behaviours and linguistic complexity. Language Learning, 67(1), 208-241. https://doi.org/10.1111/lang.12205.

Rohman, D. G. (1965). Pre-writing the stage of discovery in the writing process. College Composition and Communication, 16(2), 106-112. https://doi.org/10.2307/354885. 
Sasaki, M. (2000). Toward an empirical model of EFL writing processes: An exploratory study. Journal of Second Language Writing, 9(3), 259-291. https://doi.org/10.1016/S1060-3743(00)00028-X.

Schilperoord, J. (2002). On the cognitive status of pauses in discourse production. In T. Olive, \& C. M. Levy (Eds.), Contemporary tools and techniques for studying writing, (pp. 59-85). Dordrecht: Springer. https://doi.org/10.1007/978-94-01 0-0468-8_4

Schumacher, G. M., Klare, G. R., Cronin, F. C., \& Moses, J. D. (1984). Cognitive activities of beginning and advanced college writers: A pausal analysis. Research in the Teaching of English, 18(2), 169-187.

Spelman Miller, K. (2000a). Academic writers online: Investigating pausing in the production of text. Language Teaching Research, 4(2), 123-148. https://doi.org/10.1191/136216800675510135.

Spelman Miller, K. (2000b). Writing online: Temporal features of first and second language written text production. Doctoral dissertation. University of Reading.

Spelman Miller, K., Lindgren, E., \& Sullivan, K. P. H. (2008). The psycholinguistic dimension in second language writing: Opportunities for research and pedagogy using computer keystroke logging. TESOL Quarterly, 42(3), 433-454. https://doi. org/10.1002/j.1545-7249.2008.tb00140.x.

Van Waes, L., Leijten, M., Pauwaert, T., \& Van Horenbeeck, E. (2019). A multilingual copy task: Measuring typing and motor skills in writing with Inputlog. Journal of Open Research Software, 7(1), 1-8.

Van Waes, L., \& Schellens, P. J. (2003). Writing profiles: The effect of the writing mode on pausing and revision patterns of experienced writers. Journal of Pragmatics, 35(6), 829-853. https://doi.org/10.1016/50378-2166(02)00121-2.

Vandermeulen, N., Leijten, M., \& Van Waes, L. (2020). Reporting writing process feedback in the classroom using keystroke logging data to reflect on writing processes. Journal of Writing Research, 12(1), 109-139. https://doi.org/10.17239/jowr-202 0.12.01.05.

Wang, F., Chen, X., \& Fang, W. (2011). Integrating cell phones into a Chinese high school EFL classroom: Students' attitudes, technological readiness, and perceived learning. Journal of Educational Technology Development and Exchange, 4(1), 91102.

Wengelin, Å. (2006). Examining pauses in writing: Theory, methods and empirical data. In K. P. H. Sullivan, \& E. Lindgren (Eds.), Computer keystroke logging and writing: Methods and applications, studies in writing, (vol. 18, pp. 107-130). Oxford: Elsevier

Wengelin, A.., Torrance, M., Holmqvist, K., Simpson, S., Galbraith, D., Johansson, V., \& Johansson, R. (2009). Combined eyetracking and keystroke-logging methods for studying cognitive processes in text production. Behavior Research Methods, 41(2), 337-351. https://doi.org/10.3758/BRM.41.2.337.

Witte, S. P., \& Faigley, L. (1981). Coherence, cohesion, and writing quality. College Composition and Communication, 32(2), 189204. https://doi.org/10.2307/356693

Xu, C. (2011). Shuru rizhi he pinmu luxiang de jiaocha yunyong—Jisuanji fuzhu eryu xiezuo guocheng yanjiu xinshiye [integrating Inputlog and screen recorder: New research insights into the EFL writing process]. Waiyu Dianhua Jiaoxue [computer-assisted foreign language education], 5, 21-25.

$\mathrm{Xu}, \mathrm{C}$. (2018). Understanding online revisions in L2 writing: A computer keystroke-log perspective. System, 78, 104-114. https://doi.org/10.1016/.jsystem.2018.08.007.

Xu, C. (2021). Xiezuo tingdun shijiaoxia de zhongguo yingyu xuexizhe jixuwen he yilunwen xiezuo renzhi jiagong guocheng yanjiu [a study on the cognitive processing of Chinese English learners' narrative and argumentative writing from the perspective of writing pauses]. Waiyu Jiaoxue [Foreign Language Education], 42(1), 35-40.

Xu, C., \& Ding, Y. (2014). An exploratory study of pauses in computer-assisted EFL writing. Language, Learning and Technology, 18(3), 80-96.

Xu, C., \& Qi, Y. (2017). Analysing pauses in computer-assisted EFL writing: A computer-keystroke-log perspective. Journal of Educational Technology \& Society, 20(4), 24-34.

$X u, C$. (2017). Xiezuo tingdun shijiao xia de eryu xiezuo guocheng yanjiu [A temporal investigation of $L 2$ writing process: a pausological perspective]. Waiyu Jiaoxue yu Yanjiu [Foreign Language Teaching and Research], 49(4), 583-595.

Yang, Y. (2010). Students' reflection on online self-correction and peer review to improve writing. Computers \& Education, 55(3), 1202-1210. https://doi.org/10.1016/j.compedu.2010.05.017.

Zarrabi, F., \& Bozorgian, H. (2020). EFL students' cognitive performance during argumentative essay writing: A log-file data analysis. Computers and Composition, 55, 102546. https://doi.org/10.1016/j.compcom.2020.102546.

\section{Publisher's Note}

Springer Nature remains neutral with regard to jurisdictional claims in published maps and institutional affiliations.

\section{Submit your manuscript to a SpringerOpen ${ }^{\circ}$ journal and benefit from:}

- Convenient online submission

- Rigorous peer review

- Open access: articles freely available online

- High visibility within the field

- Retaining the copyright to your article

Submit your next manuscript at $\boldsymbol{\nabla}$ springeropen.com 